\title{
Neurocognitive Stimulation Game: Serious Game for Neurocognitive Stimulation and Assessment
}

\author{
João Costa ${ }^{1(\bowtie)}$, Jorge Neto ${ }^{1}$, Ricardo Alves ${ }^{2}$, Paula Escudeiro ${ }^{1,2}$, \\ and Nuno Escudeiro ${ }^{1,3}$ \\ ${ }^{1}$ Instituto Superior de Engenharia do Porto, Porto, Portugal \\ \{1110783,1110156,pmo,nfe\}@isep.ipp.pt \\ ${ }^{2}$ Games Interaction and Learning Technologies - GILT, Porto, Portugal \\ ricalves03@gmail.com \\ ${ }^{3}$ Laboratory of Artificial Intelligence and Decision Support, \\ INESC TEC, Porto, Portugal
}

\begin{abstract}
The ageing process is naturally accompanied by changes in people's cognitive processes. The European population ageing is a challenge for the European social policy and for the mental health professionals. New technologies can play an important role in the neurocognitive stimulation area as they possess characteristics that might reduce the anxiety levels of patients participating in neurocognitive stimulation or assessment programs. In particular, serious games provide a setting that can be explored to improve the easy access to neurocognitive stimulation and assessment, regardless of place and time, at a lower cost then traditional approaches. This paper presents a serious game aiming to analyse neurocognitive deficits and stimulate the players' deficitary neurocognitive processes. This game is built on top of sound neurocognitive psychotherapy for adults, mainly addressing the cognitive processes of attention and memory. The game will simulate real world scenarios, allowing a better generalization process due to ecological validity.
\end{abstract}

Keywords: Active ageing · Neurocognitive deficits · Neurocognitive stimulation $\cdot$ Assessment $\cdot$ Serious games $\cdot$ Unity $\cdot$ DDA

\section{Introduction}

With the increasing number of the elderly in our population, it is possible to verify the consequent increase of the cognitive decline incidence. Neurocognitive stimulation has been a highly approached research area for the past years, as it offers new opportunities for people with cognitive impairments. Several neurocognitive stimulation programs are implemented in medical context, with the aim of slowing down cognitive decline [1] and, therefore, improving the life quality of the patients. However, these programs

J. Costa, J. Neto, R. Alves contributed equally to this work.

(C) ICST Institute for Computer Sciences, Social Informatics and Telecommunications Engineering 2017

C. Vaz de Carvalho et al. (Eds.): SGAMES 2016, LNICST 176, pp. 74-81, 2017.

DOI: 10.1007/978-3-319-51055-2_10 
have some limitations that might compromise the desired impact on the individual's quotidian. These limitations involve, for example, the lack of ecological validity and patients' low motivation, due to the high emotional pressure they feel on following these neurocognitive stimulation programs and assessments [3]. Ecological validity can be seen as a transitional phenomenon, which analyses the current behavior, within specific environments related to the real world, by using discrete and reliable research methods [2].

The use of serious games is turning into a remarkable resource, as it offers computerized alternatives to neurocognitive stimulation and assessment programs. By focusing our efforts on reducing the impact of the above-mentioned limitations, i.e., reinforcing the need for ecological validity and adapt the tasks' difficulty levels, there is a high probability that the results obtained from a serious game's neurocognitive stimulation program can show positive results $[4,5]$.

Although the few projects that use serious games try to solve these problems, none of them covers efficiently the core aspects, such as ecological validity, that we address. Current approaches usually focus on only computerizing the assessments and do not always consider the content of the program itself. In addition, there is no special attention given to the users' interaction.

Our methodology consists on developing a serious game, called SynapseToLife, which focus on the neurocognitive stimulation of the players, by making them perform several tasks, immersed in well-known scenarios, thus strengthening ecological validity. More importantly, the serious game will create a group of familiar daily life scenarios (e.g. kitchen) to the user, in order to allow an easier transfer of the stimulated cognitive abilities into the users' quotidian, given the ecological validity variable that we aim to approach $[3,6]$.

\section{Problem Description}

With the increasing number of the elderly and the consequent incidence of cognitive decline associated, it becomes important to invest in mental health, to minimize the social and economic impacts of this phenomenon, promoting active ageing.

Since the neurocognitive deficits may be present about 20 years before the clinical diagnosis on dementias, such as the Alzheimer disease [7], it is necessary to develop more effective and motivating strategies of monitoring and stimulating people's cognitive abilities, allowing them to follow an healthier life style [8]. SynapseToLife will be able to perform an early intervention, which is of major importance in order to slow down possible pre-clinical manifestations of neurocognitive [9] deficits, which, consequently, will contribute for public health's cost reduction [10].

\subsection{State of the Art}

Serious games allow the monitoring and presentation of stimulus, capable of motivating the user [11] and which show a greater accessibility [12], presenting positive results [13]. 
The use of serious games has been increasingly referenced as an important resource for psychological assessment and intervention [14]. Showing positive results in multiple domains, such as prevention [15], rehabilitation [14], neurocognitive stimulation [16], assessment [17] and monitoring neurocognitive changes [18], leading to beneficial changes, when it comes to brain plasticity [19], changing the brain's structure [20] and facilitating the impact of neurocognitive stimulation on everyday functioning [6]. However, the use of serious games, with older people, is still in an early phase [21] and there is little information when it comes to the impact of these programs on the users' quotidian activities and on their quality life [22].

There are several available games in the market, which aim neurocognitive stimulation [23, 24]. These, however, are not specifically developed to target a certain population and, in most cases, are not supported by robust studies on ecological validity. Although there are empirical evidences of neurocognitive improvements, several games do not evaluate the impact of a serious game in patients' daily life and do not offer content, which benefits the generalization process of, trained tasks, to their daily reality.

\section{Proposed Solution}

In this work, we propose to develop the ACT-Age platform, which includes the serious game SynapseToLife, aiming to promote neurocognitive stimulation and assessment. The serious game will enable an easier transfer of the neurocognitive stimulation results to users' quotidian activities, by simulating real life scenarios and users' interactions with them. This will be supported by the ecological validity concept, previously outlined, and which will play a significant role when it comes to reduce the users' anxiety levels, consequently enhancing their motivation, while being cognitively stimulated, and increasing the efficiency of the neurocognitive stimulation's results.

Throughout the game, tasks, adapted from the neurocognitive stimulation programs traditionally implemented, will be presented to the users along with a calculated difficulty level. The purpose of the game's tasks, is to simulate real life situations where users need to evoke their cognitive processes. A dynamic difficulty adjustment (DDA) component will also be an essential tool to develop, as it is a powerful expert control system, capable of studying and interpreting users' performances, throughout the game, and adapting (controlling) the game and tasks' difficulty according to the users' cognitive capabilities, directly assisting each one of them.

\subsection{Main Components}

SynapseToLife is organized in four different scenarios, where each will simulate real world situations, as the user will need to go through them, while performing the intended stimulation tasks. By structuring the game flow in mini games, adapted to real life situations or problems, users will hardly percept they are performing stimulation tasks and will only worry about having fun by completing these random tasks. 
Another important component to be developed is the DDA system, as it is crucial when it comes to adapt the mini games' difficulty to the user's cognitive ability. Its job involves analyzing the player's performance and assure that he or she keeps relaxed and in a concentrated state of mind, by constantly presenting challenges and rewarding them accordingly (game flow). Shortly, the DDA allows the automatic readjustment of the game's difficulty, based on the player's performance results. These results, along with all users' interactions while playing the game, will be recorded in a centralized server. Later on, the expert will analyze these same results, by accessing this server. These actions are transformed into useful and careful information and, more specifically, in data that the expert needs in order to study the player's performance, such as the number of right answers, the number of attempts, the response times or the cognitive processes approached (e.g. memory).

Lastly, and before the game starts, a diagnostic test will be presented to the user with the aim of setting a baseline, enabling posterior comparisons and analyzing the evolution of the players' cognitive status. This way, it is possible to understand if neurocognitive stimulation occurred and if the game itself presents all the tools needed to perform this stimulation.

\subsection{Game Structure}

Figure 1 presents the relationship between the business concept (cognitive stimulation) and the project development. With this scheme, it becomes easier to visualize the interaction between the user and the expert.

Players will need to complete the mini games and a diagnostic test, which will be better approached further on. The final results are reported to the expert, to perform the diagnosis. Both the IT staff and the expert will provide assistance to the players, in case they have doubts related to the activities they will need to perform. In Fig. 2, it is possible to understand, more specifically, the game flow, which consists on the game's life cycle.

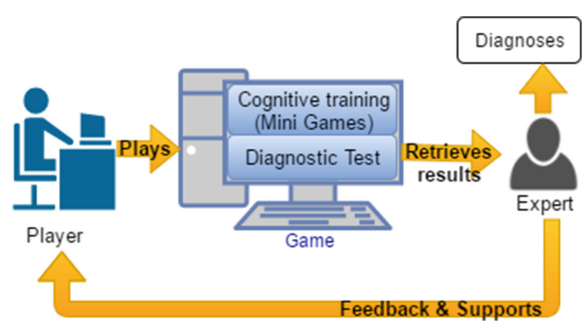

Fig. 1. Architectural model.

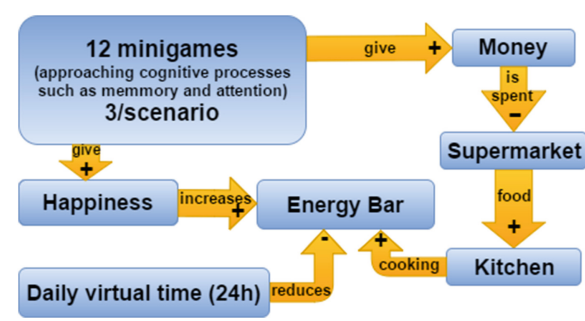

Fig. 2. Game flow 


\subsection{Game Scenarios}

All scenarios, presented in Fig. 3, will be developed in 3D, by using the Unity3D platform. The tridimensional model was chosen due to the ecological validity factor. The more detail the game presents, the more the players become concentrated and the easier it is for them to understand the logic behind the tasks they perform throughout the game, which mainly focus on certain cognitive processes, like memory and attention. Each scenario is responsible for asking the completion of tasks related to that specific scenario. For example, in the Kitchen scenario, the player only performs kitchen related tasks, such as cooking, promoting stimuli on specific cognitive processes. The same applies to the other scenarios and this is the base of ecological validity, i.e., answer questions or performing tasks that are inserted in a given context and that can actually happen in a real life situation (Fig. 4).

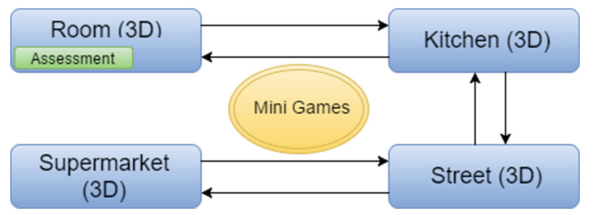

Fig. 3. Game scenarios.

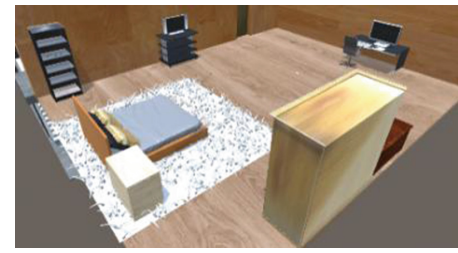

Fig. 4. Scenario example.

\section{Expected Outcomes}

After the project development phase, the users and the serious game will be both evaluated, in different ways. The game must be able to retrieve, from the users' interaction with the game, information needed for the expert, in order for him to draw his conclusions. The serious game will also need to be able to analyze the players' responses throughout the game, since the tasks' difficulty levels must be adapted according to the user needs and since it is essential for us to study these same responses and observe their interaction. During this assessment phase, users' responses will be assessed and carefully analyzed, i.e., the extracted and quantified results, from the neurocognitive stimulation program performed, will be studied.

\subsection{Output Model}

The players' actions are directly connected to their performance, i.e., there is a set of features that help us understand how the user performance during the game or how this same user is dealing with it. The variables (e.g. right answers) together, form this output model so that the expert can analyze the players' cognitive ability in the best way possible. The output model, in turn, will be allocated in the available server.

However, the game has an error-free structure, which means that the player will not advance to the next phase without understanding what he or she did wrong and without 
knowing how can the current problem must be solved. This way, the neurocognitive stimulation has more impact on the player, as the probability of him solving an equal problem, in the future, increases.

\subsection{Assessment Plan}

The assessment will be based on a study with a quasi-experimental design, where an independent variable (cognitive training) is manipulated, verifying its effect on dependent variables (e.g. attention). Taking this into account, and shortly, the assessment process consists in the following steps:

- Interview and select the most suitable participants for the experiment, using the previously defined restrictions.

- Compare the pre-assessment results with the post-assessment results, analyzing all data obtained from the game and the neurocognitive assessment;

- Post-Post assess the participants after a few weeks or months, in order to confirm if there was retention of the cognitive skills acquired.

After the project development, six weeks will be reserved for this final assessment process, conducted by the expert and supported by the IT staff, in order to give a scientific answer to the work accomplished.

\subsection{Main Contributions}

Overall, the outcomes expected are based on the cognitive assessment itself, i.e., the game should present better results when compared with classic neurocognitive stimulation programs. The project ought to also show improvements on the interaction between the user and the computer, as efforts will be made related to this situation. Furthermore, it is essential making the system able to be sensitive to the players' interaction with the game's environment (e.g. verify if the tasks are having the correct reactions) and adapt its difficulty accordingly. We also expect to confirm that our serious game has ecological validity, since it has a preponderant role in the project's main purpose.

\section{Conclusions}

The serious game proposed in this work aims to overcome the limitations of current solutions addressing active ageing. It is based on a set of scenarios simulating familiar environments and everyday activities of an individual's daily life. This allows stimulating users' cognitive abilities and transferring the stimulation results to the tasks normally performed during their quotidian. The user interaction with the game, while playing it for stimulation, will be monitored and recorded in a cognitive model to allow guiding the effective stimulation towards deficitary cognitive processes. This model will also allow the neuropsychological profile monitoring and an effective intervention 
with ecological impact. We expect this serious game to become a robust tool being able to study, interpret and stimulate users' neurocognitive processes.

We aim to provide our future players a welcoming environment, by carefully analyzing which content is necessary to insert inside the game, in order to promote ecological validity, which is our main focus during the development phase, and by developing a tutorial which will help the player to get acquainted to the gameplay. Moreover, the DDA component must be able to analyze every situation possible and every user interaction throughout the game. It is also necessary to consider the interaction itself.

Thus, we seek to develop a solid serious game, in a technological and scientific point of view, which will promote the development of the knowledge necessary for the implementation of this technology in new markets with potential growth, aiming several domains, such as health, well-being, ageing and social inclusion. This project also seeks to give answers to questions based on the implementation, experimentation challenges, quality control in application domains and impact on users' quality life. These scientific evidences will allow a safer investment from the digital games' industry professionals in developing the "serious" market of games. Another important activity intends to disclose the project and its results.

\section{References}

1. Glisky, E.: Changes in cognitive function in human aging. In: Riddle, D.R. (ed.) Brain Aging: Models, Methods, and Mechanisms. CRC Press, Boca Raton (2007)

2. Rizzo, A., Kim, G.J.: A SWOT analysis of the field of VR rehabilitation and therapy. Presence Teleoper. Virtual Environ. 14, 119-146 (2005). doi:10.1162/1054746053967094

3. Segal, R., Bhatia, M., Drapeau, M.: Therapist's perception of benefits and costs of using virtual reality treatments. Cyberpsychology Behav. Soc. Networking 14(1-2), 29-34 (2011)

4. Fissler, P., Kolassa, I.T., Schrader, C.: Educational games for brain health: revealing their unexplored potential through a neurocognitive approach. Front. Psychol. 6, 1056 (2015). doi:10.3389/fpsyg.2015.01056

5. Powers, K., Brooks, P., Aldrich, N., Palladino, M., Alfieri, L.: Effects of video-game play on information processing: a meta-analytic investigation. Psychon. Bull. Rev. 20, 1055-1079 (2013). doi:10.3758/s13423-013-0418-z

6. Spector, A., Orrell, M., Woods, B.: Cognitive stimulation to improve cognitive functioning in people with dementia. Int. J. Geriatr. Psychiatry 25(12), 1253-1258 (2010)

7. Rajan, K., Wilson, R., Weuve, J.: Cognitive impairment 18 years before clinical diagnosis of Alzheimer disease dementia. Neurology published online 24 June 2015. doi:10.1212/WNL. 0000000000001774

8. Mcguire, A.M., Anderson, D.J., Fulbrook, P.: Perceived barriers to healthy lifestyle activities in midlife and older Australian women with type 2 diabetes. Collegian (2013). doi:10.1016/j. colegn.2013.07.001

9. American Psychiatric Association: Diagnostic and statistical manual of mental disorders, 5th edn. American Psychiatric Publishing, Arlington (2013)

10. Direção-Geral da Saúde: Portugal Saúde Mental - 2013. Programa Nacional para a Saúde Mental. Obtido em 7 de 2 de 2015, de Direcção-Geral da Saúde (2013). http://www.dgs.pt/ publicacoes 
11. Manera, V., Petit, P., Derreumaux, A., Orvieto, E., et al.: 'Kitchen and cooking', a serious game for mild cognitive impairment and Alzheimer's disease: a pilot study. Frontiers Aging Neurosci. 7, 24 (2015). doi:10.3389/fnagi.2015.00024

12. Krebs, P., Prochaska, J.O., Rossi, J.S.: A meta-analysis of computer-tailored interventions for health behavior change. Prev. Med. 51, 214-221 (2010)

13. Connolly, T., Boyle, E., MacArthur, E., et al.: A systematic literature review of empirical evidence on computer games and serious games. Comput. Educ. 59(2), 661-686 (2012). doi:10.1016/j.compedu.2012.03.004

14. Robert, P.H., König, A., Amieva, H., et al.: Recommendations for the use of serious games in people with Alzheimer's disease, related disorders and frailty. Frontiers Aging Neurosci. 6, 54 (2014). doi:10.3389/fnagi.2014.00054

15. Wiemeyer, J., Kliem, A.: Serious games in prevention and rehabilitation - a new panacea for elderly people? Eur. Rev. Aging Phys. Act. 9, 41-50 (2012). doi:10.1007/s11556-011-0093-x

16. Nouchi, R., Taki, Y., Takeuchi, H., Hashizume, H., Akitsuki, Y., et al.: Brain training game improves executive functions and processing speed in the elderly: a randomized controlled trial. PLoS ONE 7(1), e29676 (2012). doi:10.1371/journal.pone.0029676

17. Anguera, J.A., Boccanfuso, J., Rintoul, J.L., Al-Hashimi, O., et al.: Video game training enhances cognitive control in older adults. Nature 501, 97-102 (2013). doi:10.1038/ nature 12486

18. Tarnana, I., Papagiannopoulos, S., Kazis, D., Wiederhold, M., Widerhold, B., Tsolak, M.: Reliability of a novel serious game using dual-task gait profiles to early characterize aMCI. Front. Aging Neurosci. 7(50), 1-15 (2015). doi:10.3389/fnagi.2015.00050

19. Lövdén, M., Bäckman, L., Lindenberger, U., Schaefer, S., Schmiedek, F.: A theoretical framework for the study of adult cognitive plasticity. Psychol. Bull. 136, 659-676 (2010). doi:10.1037/a0020080

20. Kühn, S., Gallinat, J.: Amount of lifetime video gaming is positively associated with entorhinal, hippocampal and occipital volume. Mol. Psychiatry 19, 842-847 (2014). doi:10. 1038/mp.2013.100

21. Tarnanas, I., Tsolaki, M., Nef, T., et al.: Can a novel computerized cognitive screening test provide additional information for early detection of Alzheimer's disease? Alzheimers Dement. 10, 790-798 (2014). doi:10.1016/j.jalz.2014.01.002

22. Kazmi, S., Ugail, H., Valerie, L., Palmer, I.: Interactive digital serious games for the assessment, rehabilitation, and prediction of dementia. Int. J. Comput. Games Technol. 2014, 1-11 (2014). doi:10.1155/2014/701565. Article ID 701565

23. McCallum, S., Boletsis, C.: Dementia games: a literature review of dementia-related serious games. In: Ma, M., Oliveira, M.F., Petersen, S., Hauge, J.B. (eds.) SGDA 2013. LNCS, vol. 8101, pp. 15-27. Springer, Heidelberg (2013). doi:10.1007/978-3-642-40790-1_2

24. Arambarri, J., Torre, I., Coronado, M., Álvarez, I.: Investigating the potential market of a serious game for training of alzheimer's caregivers in a Northern Spain region. Int. J. Serious Games 1(4), 75-79 (2014). doi:10.17083/ijsg.v1i4.36. ISSN: 2384-8766 